\title{
OTIMIZAÇÃO VIA METODOLOGIA DE SUPERFÍCIE DE RESPOSTAS DOS PARÂMETROS TECNOLÓGICOS PARA PRODUÇÃO DE FRUTA ESTRUTURADA E DESIDRATADA A PARTIR DE POLPA CONCENTRADA DE MAMÃO ${ }^{1}$
}

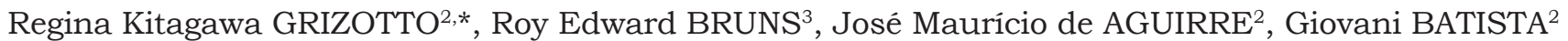

\begin{abstract}
RESUMO
O objetivo deste trabalho foi investigar os parâmetros tecnológicos relevantes no processo de estruturação de polpa concentrada de mamão (16 $6^{\circ}$ Brix), utilizando um delineamento experimental associado a Metodologia de Superficie de Respostas. Os resultados do planejamento composto central utilizado mostraram que as variáveis pectina $\left(\mathrm{x}_{1}\right)$ e alginato $\left(\mathrm{x}_{2}\right)$ foram estatisticamente significativas para aumentar a firmeza de fruta estrutura de mamão. Foram realizados experimentos para confirmar as previsões do modelo matemático proposto para firmeza. As frutas estruturadas obtidas com os menores niveis de pectina $(7 \mathrm{~g} / \mathrm{kg})$ e alginato $(7 \mathrm{~g} / \mathrm{kg})$ apresentaram firmeza elevada, em torno de $3,2 \mathrm{~kg}$, cerca de 2,4 vezes maior que o valor previsto pelo modelo, provavelmente devido a variações no $\mathrm{pH}$ da polpa de mamão. Os resultados mostraram ser possivel produzir fruta estruturada com teor elevado de polpa de mamão concentrada $(672 \mathrm{~g} / \mathrm{kg})$ e quantidade mínima de sacarose $(364 \mathrm{~g} / \mathrm{kg})$. O glicerol $\left(\mathrm{x}_{3}\right)$ na concentração de $100 \mathrm{~g} / \mathrm{kg}$ suprimiu a atividade de água dos géis de polpa concentrada de mamão para 0,922 . A secagem $\left(60^{\circ} \mathrm{C} / 6 \mathrm{~h}\right)$ sob vácuo $(0,8 \mathrm{kgf} / \mathrm{kg})$ das frutas estruturadas minimizou o problema de adesividade superficial das frutas estruturadas e, concomitantemente, promoveu redução na atividade de água, para niveis de umidade intermediária, em torno de 0,860.

Palavras-chave: fruta estruturada; polpa de mamão; hidrocolóides; otimização; secagem.
\end{abstract}

\section{SUMMARY}

OPTIMIZATION BY THE SURFACE RESPONSE METHODOLOGY OF TECHNOLOGICAL PARAMETERS FOR THE PRODUCTION OF RESTRUCTURED AND DRIED FRUIT MADE FROM CONCENTRATED PAPAYA PULP. The objective of this study was to investigate the relevant technological parameters of the manufacturing process of restructured fruit made from concentrated papaya pulp $\left(16^{\circ} \mathrm{Brix}\right)$ using an experimental delineation method associated with the Surface Response Methodology. The results of the Central Composed Design utilized show that the effect of the variables pectin $\left(\mathrm{x}_{1}\right)$ and alginate $\left(\mathrm{x}_{2}\right)$ on the increase in firmness of the papaya fruit restructured is statistically significant. A series of experiments was conducted to confirm the values predicted by the mathematical model proposed for firmness. The restructured fruits produced with the lowest addition levels of pectin $(7 \mathrm{~g} / \mathrm{kg})$ and alginate $(7 \mathrm{~g} / \mathrm{kg})$ exhibited high firmness values of about $3.2 \mathrm{~kg}$. These values were approximately 2.4 times greater than the values for firmness predicted by the model, probably due to variations in the $\mathrm{pH}$ of the papaya pulps used in the experiments. The results showed that it is possible to manufacture restructured fruit using high levels of concentrated papaya pulp $(672 \mathrm{~g} / \mathrm{kg})$ and minimum amounts of sucrose $(364 \mathrm{~g} / \mathrm{kg})$. Glycerol $\left(\mathrm{x}_{3}\right)$ used at the concentration of $100 \mathrm{~g} / \mathrm{kg}$ reduced the level of water activity of the concentrated papaya pulp gels to 0.922 . Vacuum drying $\left(60^{\circ} \mathrm{C} / 6 \mathrm{~h} ; 0.8 \mathrm{kgf} / \mathrm{kg}\right)$ of the restructured fruits minimized the problem of surface adhesion and, at the same time, lowered water activity to intermediate levels of about 0.860 .

Keywords: restructured fruit; papaya pulp; hydrocolloids; optimization; drying.

\section{1 - INTRODUÇÃO}

O Brasil é considerado o maior produtor mundial de mamão, tendo participado com cerca de 1,4 milhões de toneladas anuais em 2001. Destes, somente uma pequena porcentagem $(1,5 \%)$ destinaram-se à exportação e o restante, consumido internamente [1]. A maior parte da produção nacional é consumida na forma in natura e apenas uma pequena parte é industrializada [12].

A industrialização do mamão constitui-se na melhor opção para minimizar as pesadas perdas que ocorrem por ocasião das grandes safras, quando as frutas alcançam preços muito baixos no mercado da fruta fres-

\footnotetext{
Recebido para publicação em 06/02/2004. Aceito para publicação em 01/02/2005 (001286).

2. Centro de Tecnologia de Hortifrutícolas, Instituto de Tecnologia de Alimentos - ITAL. Av. Brasil, 2880, Jd. Chapadão, Campinas, SP - CEP 13073-001 - C.P. 139 - e-mail: regina@ital.org.br

3. Instituto de Quimica, Universidade Estadual de Campinas, UNICAMP. Cidade Universitária "Zeferino Vaz", Barão Geraldo - Campinas, SP CEP 13083-970 - C.P.6154.

* A quem a correspondência deve ser enviada.
}

ca. Os produtos que podem ser obtidos da industrialização do mamão incluem purê (congelado ou pasteurizado), néctar, sucos, mamão em calda, cristalizado, geléias e, principalmente, como ingrediente para salada de frutas tropicais, além da papaína extraída do látex [12]. Há, entretanto, necessidade de diversificar os produtos industrializados obtidos do purê ou polpa de mamão, oferecendo produtos inovadores, convenientes e de maior valor agregado.

O presente trabalho propõe o uso de misturas de hidrocolóides (alginato e pectina de baixa metoxilação) como agentes de união, para facilitar o corte, favorecer a retenção de umidade, fatores esses que contribuirão para delinear nova textura em produto estruturado contendo quantidades elevadas de polpa concentrada de mamão. Esses produtos podem ser utilizados na formulação de produtos de confeitaria ou alimentos congelados, ou consumidos na forma que se apresentam, como um confeito, similarmente às barras de frutas (fruit bar) também conhecidas como "couro" de frutas (leather fruit) [21]. O "couro" de frutas é um produto de confeitaria resultante da superposição de lâminas de purê de frutas desidratadas, de textura coriácea largamente consumida na Índia, segundo Raab e Oehler citado por VIJAYANAND et al.[21]. 
Os procedimentos de estruturação descritos por diversos pesquisadores $[4,8,10,13,14,15,16,17,19]$ ainda apresentam limitações. É consenso que a adição de quantidade relativamente elevada de polpa de fruta, tende a enfraquecer o gel, e o produto final apresenta textura pastosa e de qualidade inadequada $[14,15]$. O efeito da adição de polpa nas propriedades mecânicas do gel depende do $\mathrm{pH}$ da polpa $[10,13,14]$ e do tipo de polpa $[11,16]$, entre outros fatores.

A sinérese é um problema freqüentemente verificado em géis de polpa de frutas, resultado da liberação da água retida na matriz do gel $[7,13,14]$. A umidade superficial das frutas estruturadas provocada pela exudação de líquidos, proporciona condições para o desenvolvimento microbiano, e a adesividade superficial dificulta a sua aplicação como um produto pronto para consumo. A intensidade da sinérese pode ser utilizada como um indicador da estabilidade do gel [7]. A adição de sacarose melhora a textura dos géis quando em concentrações entre 300 a $400 \mathrm{~g} / \mathrm{kg}[7,13,16]$ provavelmente devido à ação nas propriedades do solvente reduzindo a interação polímero-solvente e, promovendo a atração polímero-polímero [16]. Uma outra alternativa, ainda pouco explorada, para minimizar o problema da sinérese em produtos estruturados, seria a remoção da umidade por meios físicos, como a secagem. A secagem, além de reduzir o nível de água livre, essencial para a estabilização microbiológica do produto, também contribui para melhorar a textura do produto [18]. A textura é considerada o atributo organoléptico que mais influencia a aceitabilidade de produtos processados [2]. Entretanto, há carência de informações sobre os procedimentos tecnológicos empregados, a correta formulação dos ingredientes usados na elaboração de produtos estruturados e o efeito da secagem na textura dos produtos estruturados.

A Metodologia de Superficie de Respostas (MSR) [3, 5] é uma ferramenta eficiente para otimizar as propriedades de alimentos processados. Baseia-se na variação simultânea de vários fatores (variáveis independentes), previamente selecionados por sua influência nas propriedades do processo (variáveis dependentes ou respostas). Utilizando técnicas matemáticas e estatísticas, os resultados experimentais indicam uma combinação de níveis dos fatores dentro de uma região ótima.

O objetivo deste trabalho foi investigar os parâmetros relevantes para a estruturação de polpa concentrada de mamão $\left(16^{\circ} \mathrm{Brix}\right)$, avaliando o efeito da combinação de alginato, pectina de baixa metoxilação e glicerol nas características do gel de fruta contendo entre 640 a $690 \mathrm{~g} / \mathrm{kg}$ de polpa concentrada de mamão. A formulação e o processamento para preparar as frutas estruturadas com elevado teor de sólidos $\left(50^{\circ} \mathrm{Brix}\right)$ foram otimizados em relação as propriedades do produto final em termos de firmeza, $\mathrm{pH}$, sólidos solúveis ( $\left.{ }^{\circ} \mathrm{Brix}\right)$ e atividade de água, utilizando delineamento experimental para obter as superficies de respostas. $O$ efeito da secagem $\left(60^{\circ} \mathrm{C} / 6 \mathrm{~h}\right)$ sob vácuo $\left(0,8 \mathrm{kgf} / \mathrm{cm}^{2}\right)$ foi avaliado em frutas estruturadas de polpa concentrada de ma- mão produzidas de acordo com os parâmetros otimizadas pela MSR.

\section{2 - MATERIAIS E MÉTODOS}

\section{1 - Materiais}

A polpa de mamão ( $\mathrm{pH} 4,19 ; 10,2^{\circ}$ Brix) foi concentrada em tacho encamisado (Groen MGF Co, EUA) de 30 litros de capacidade, operando a $40-45^{\circ} \mathrm{C}$ sob vácuo $\left(0,8 \mathrm{kgf} / \mathrm{cm}^{2}\right)$. A polpa concentrada obtida $(\mathrm{pH} 4,00$; $16^{\circ}$ Brix) foi utilizada como matéria-prima neste trabalho.

Os hidrocolóides utilizados foram pectina de baixa metoxilação com 33 a 37\% de esterificação (Danisco Cultor México, México) e alginato de sódio (Danisco Ingredients Landerneau, França) ambos de grau alimentício. Os coadjuvantes tecnológicos utilizados no processo de estruturação de polpa de mamão concentrada foram fosfato de cálcio bibásico anidro p.a. $\left(\mathrm{CaHPO}_{4}\right)$ (Synth, Brasil), glucona-delta-lactona (GDL) (ADM, USA), glicerol $\left(\mathrm{C}_{3} \mathrm{H}_{5}(\mathrm{OH})_{5}\right)$ p.a. (Synth, Brasil) e sacarose refinada comercial (União, Brasil).

\section{2 - Estruturação de polpas concentradas de fru- tas}

As frutas estruturadas foram preparadas de acordo com procedimento apresentado na Figura 1, utilizandose proporções combinadas de alginato, pectina e glicerol de acordo com o delineamento estatístico composto central.
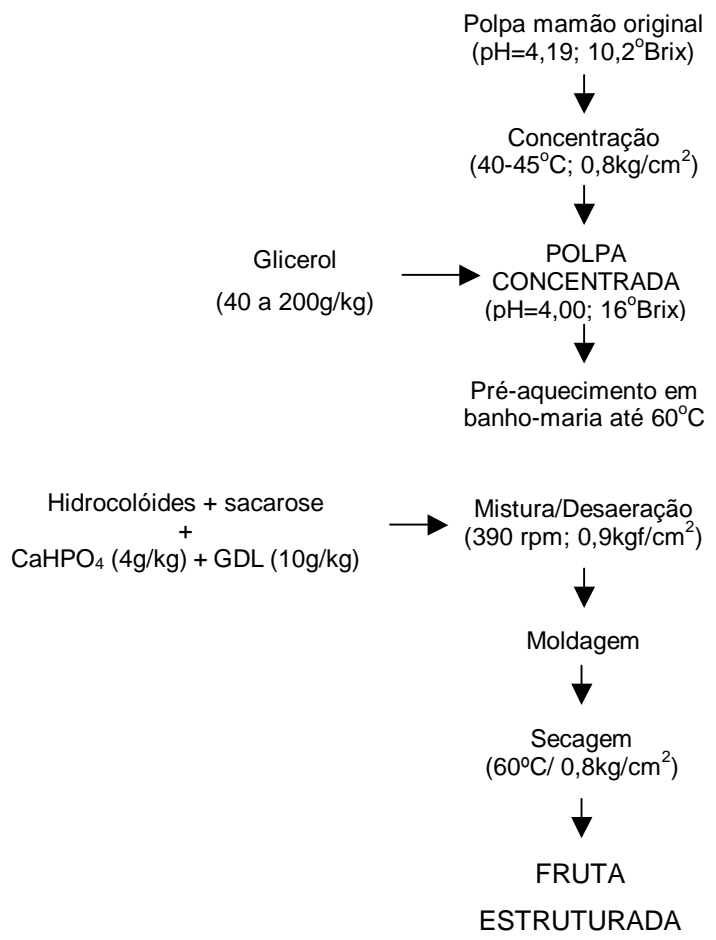

FIGURA 1. Fluxograma básico de processamento para produção de fruta estruturada de umidade intermediária, incluindo a etapa de concentração da polpa original. 
À polpa concentrada foi adicionada quantidade de glicerol variando de 40 a $200 \mathrm{~g} / \mathrm{kg}$ (Tabela 2) e, em função do teor de sólidos solúveis, calculou-se a quantidade de sacarose suficiente para elevar o teor de sólidos para $50^{\circ}$ Brix. Uma mistura seca, contendo proporções variáveis de pectina e alginato dispersa em sacarose, foi adicionada à polpa previamente aquecida a $60^{\circ} \mathrm{C}$, sob vigorosa agitação (390rpm) no Misturador-Desaerador (Cunnington \& Cooper Ltda., England), operando sob vácuo $\left(0,9 \mathrm{kgf} / \mathrm{cm}^{2}\right)$. Após dez minutos de agitação, o sistema foi aberto, liberando o vácuo, sendo adicionado simultaneamente uma suspensão de $4 \mathrm{~g} / \mathrm{kg}$ de cálcio insolúvel em $2 \mathrm{~mL}$ de água destilada, e uma solução de Glucona-Delta-Lactona $(10 \mathrm{~g} / \mathrm{kg})$, recém-preparada em $2 \mathrm{~mL}$ de água destilada. Os ingredientes foram então vigorosamente homogeneizados por mais 5 minutos. Para a moldagem das frutas estruturadas foram utilizadas placas de Petri de $50 \mathrm{~mm}$ de diâmetro e $10 \mathrm{~mm}$ de altura, com capacidade para $25 \mathrm{~g}$ de amostra, aproximadamente. As frutas assim estruturadas foram mantidas sob refrigeração a $10^{\circ} \mathrm{C}$ durante $24 \mathrm{~h}$ para completar a geleificação. Após terem sido retiradas dos moldes, as frutas estruturadas foram secionadas no formato de cilindro sólido, volume aproximado de $2 \mathrm{~cm}^{3}$, com auxílio de dispositivo de aço inox e superfície cortante com $15 \mathrm{~mm}$ de diâmetro. As frutas estruturadas assim secionadas foram submetidas à secagem a $60^{\circ} \mathrm{C}$ sob vácuo $\left(0,8 \mathrm{kgf} / \mathrm{cm}^{2}\right)$ durante 6 horas com o intuito de reduzir a água livre do produtos e minimizar a adesividade excessiva na superficie das frutas estruturadas.

\section{3 - Medida da firmeza}

A firmeza da fruta estruturada foi medida em texturômetro TA.XT2 (Stable Micro Systems, Godalming, EUA) utilizando-se sonda cilíndrica de $35 \mathrm{~mm}$ de diâmetro, denominada $\mathrm{P} / 35$ e célula de carga de $25 \mathrm{~kg}$ conforme metodologia descrita no manual de aplicações do TA.XT2 referência SWTI/P35 [20].

\section{4 - Atividade de água, pH e sólidos solúveis}

A atividade de água experimental foi determinada em higrômetro (Aqualab, Decagon Devices Inc., USA) modelo CX-12 acoplado a um banho termostático. O valor de atividade de água experimental foi corrigido utilizando-se a equação de correção obtida da calibração do equipamento com soluções de atividade de água conhecida. $\mathrm{O} \mathrm{pH}$ foi determinado pelo método potenciométrico $\mathrm{n}^{\circ}$ 4.7.2 e sólidos solúveis por refratometria [9].

\section{5 - Delineamento experimental e modelagem}

O processo de estruturação de polpa de mamão concentrada foi otimizado pela Metodologia de Superficie de Respostas descrita por BOX, HUNTER \& HUTER [5] e BARROS NETO, SCARMINIO \& BRUNS [3].

Esta metodologia consiste de um conjunto de técnicas matemáticas e estatísticas, que permite a análise de problemas onde variáveis independentes ou fatores, controlados pelo pesquisador, influenciam uma ou mais variáveis dependentes ou respostas. São execu- tados experimentos em diferentes niveis dos fatores, selecionados de acordo com delineamento estatístico multivariado. O delineamento composto central utilizado nesse trabalho, compreende o planejamento estatístico fatorial $2^{3}$, ponto central e o delineamento em estrela. Foram executadas cinco repetições do ponto central para dar a estimativa do erro experimental. Os 19 pontos experimentais foram delineados para permitir a avaliação dos modelos linear e quadrático. O modelo determinado foi testado para falta de ajuste fazendo teste $\mathrm{F}$ através da Análise de Variância (ANOVA) e teste $t$ dos coeficientes relativos aos seus erros padrões. As respostas ou variáveis dependentes utilizadas para avaliar o produto texturizado foram firmeza $\left(\mathrm{Y}_{1}\right)$, sólidos solúveis $\left(\mathrm{Y}_{2}\right), \mathrm{pH}\left(\mathrm{Y}_{3}\right)$ e atividade de água $\left(\mathrm{Y}_{4}\right)$.

Para confirmar as previsões do modelo matemático para firmeza das frutas estruturadas, foram realizados experimentos utilizando os parâmetros otimizados via MSR.

\section{3 - RESULTADOS E DISCUSSÃO}

O procedimento de estruturação adotado neste trabalho mostrou ser possivel a produção de fruta estruturada com teor elevado de polpa concentrada de mamão $(640$ a $690 \mathrm{~g} / \mathrm{kg})$ e quantidade mínima de sacarose (340 a $420 \mathrm{~g} / \mathrm{kg}$ ). O glicerol foi o soluto utilizado como supressor da atividade de água, dentro da faixa de concentração de 40 a $200 \mathrm{~g} / \mathrm{kg}$.

A polpa de mamão não pode ser concentrada além de $16^{\circ}$ Brix, mesmo após 4,5 horas de concentração. As causas deste baixo nível de concentração não estão completamente elucidadas. Supõe-se haver deposição de substâncias pécticas ou outras, presentes na polpa de mamão, na superficie interna do tacho encamisado, diminuindo a eficiência da transmissão de calor entre o meio de aquecimento (vapor) e a polpa de mamão. As propriedades físico-químicas da polpa de mamão original e concentrada, utilizada nos experimentos de otimização são apresentados na Tabela 1.

TABELA 1. Determinações físico-químicas realizadas nas polpas de mamão original e concentrada, utilizadas nos experimentos de otimização.

\begin{tabular}{lll}
\hline \multirow{2}{*}{ Análises Físico-Químicas ${ }^{(1)}$} & \multicolumn{2}{c}{ Polpa de mamão } \\
\cline { 2 - 3 } & Original & Concentrada \\
\hline Atividade de água & $0,994 \pm 0,00$ & $0,995 \pm 0,00$ \\
$\mathrm{PH}$ & $4,19 \pm 0,00$ & $4,00 \pm 0,01$ \\
Sólidos solúveis ('Brix) & $9,6 \pm 0,3$ & $16 \pm 0,1$ \\
Umidade (g/kg) & $902 \pm 0,0$ & $822 \pm 0,0$ \\
Sólidos totais $(\mathrm{g} / \mathrm{kg})$ & $98 \pm 0,0$ & $178 \pm 0,0$
\end{tabular}

(1) Média de 3 determinações \pm desvio padrão $\left(\delta_{\mathrm{n}-1}\right) ;$ onde $\delta_{(\mathrm{n}-1)}=\mathrm{s} .(\sqrt{ } \mathrm{n})^{-1} ; \mathrm{s}^{2}=\left[\sum \mathrm{x}^{2}-\left(\sum \mathrm{x}\right)^{2} /\right.$ $\mathrm{n}] .(\mathrm{n}-1)^{-1} ; \mathrm{n} \rightarrow \mathrm{n} .^{\circ}$ de amostra.

A quantidade de sacarose utilizada neste trabalho encontra-se dentro da faixa (300 a $400 \mathrm{~g} / \mathrm{kg}$ ) sugerida para estruturar polpas de maracujá [13] e manga [14]. A 
adição de glicerol contribuiu com aumento de 15 a 60\% no teor de sólidos solúveis, possibilitando redução na quantidade de sacarose adicionada para atingir $50^{\circ} \mathrm{Brix}$.

\section{1 - Otimização do processo de estruturação via MSR}

Na Tabela 2 são apresentados os resultados de firmeza, sólidos solúveis ( $\left.{ }^{\circ} \mathrm{Brix}\right), \mathrm{pH}$ e atividade de água (Aa) determinados na fruta estruturada de polpa concentrada de mamão, obtidos dos experimentos do delineamento central composto.

TABELA 2. Resultados dos experimentos do delineamento central composto para firmeza, sólidos solúveis, $\mathrm{pH}$ e atividade de água para fruta estruturada de polpa concentrada de mamão

\begin{tabular}{|c|c|c|c|c|c|c|c|c|c|c|}
\hline \multirow{2}{*}{ Experimento } & \multicolumn{3}{|c|}{$\begin{array}{l}\text { Variáveis em unidades } \\
\text { originais }(\mathrm{g} / \mathrm{kg})\end{array}$} & \multicolumn{3}{|c|}{$\begin{array}{l}\text { Variáveis em } \\
\text { unidades } \\
\text { codificadas }^{(a)}\end{array}$} & \multicolumn{4}{|c|}{ Respostas } \\
\hline & $\begin{array}{l}\text { Pectina } \\
\text { [P] }\end{array}$ & $\begin{array}{l}\text { Alginato } \\
{[\mathrm{A}]}\end{array}$ & $\begin{array}{c}\text { Glicerol } \\
\text { [G] }\end{array}$ & $\mathrm{x} 1$ & $x^{2}$ & $\times 3$ & $\begin{array}{c}\text { Firmeza } \\
(\mathrm{g})\end{array}$ & $\begin{array}{c}\text { Sólidos } \\
\text { solúveis } \\
\left({ }^{\circ} B r i x\right)\end{array}$ & $\mathrm{pH}$ & $\begin{array}{l}\text { Atividade } \\
\text { de água }\end{array}$ \\
\hline 1 & 2 & 2 & 40 & -1 & -1 & -1 & 77,53 & 43,0 & 3,51 & 0,935 \\
\hline 2 & 8 & 2 & 40 & 1 & -1 & -1 & 504,17 & 44,1 & 3,48 & 0,935 \\
\hline 3 & 2 & 8 & 40 & -1 & 1 & -1 & 413,93 & 44,0 & 3,66 & 0,935 \\
\hline 4 & 8 & 8 & 40 & 1 & 1 & -1 & 1531,11 & 43,7 & 3,55 & 0,921 \\
\hline 5 & 2 & 2 & 160 & -1 & -1 & 1 & 128,60 & 43,6 & 3,39 & 0,912 \\
\hline 6 & 8 & 2 & 160 & 1 & -1 & 1 & 597,83 & 43,2 & 3,61 & 0,910 \\
\hline 7 & 2 & 8 & 160 & -1 & 1 & 1 & 705,10 & 44,7 & 3,65 & 0,908 \\
\hline 8 & 8 & 8 & 160 & 1 & 1 & 1 & 1431,83 & 44,3 & 3,68 & 0,907 \\
\hline 9 & 0 & 5 & 100 & $-1,68$ & 0 & 0 & 166,00 & 42,2 & 3,55 & 0,929 \\
\hline 10 & 10 & 5 & 100 & $+1,68$ & 0 & 0 & 1567,46 & 42,9 & 3,60 & 0,927 \\
\hline 11 & 5 & 0 & 100 & 0 & $-1,68$ & 0 & 286,60 & 42,4 & 3,49 & 0,927 \\
\hline 12 & 5 & 10 & 100 & 0 & $+1,68$ & 0 & 1260,50 & 43,3 & 3,64 & 0,927 \\
\hline 13 & 5 & 5 & 0 & 0 & 0 & $-1,68$ & 794,80 & 42,6 & 3,55 & 0,905 \\
\hline 14 & 5 & 5 & 200 & 0 & 0 & $+1,68$ & 533,53 & 44,3 & 3,61 & 0,948 \\
\hline 15 & 5 & 5 & 100 & 0 & 0 & 0 & 925,3 & 43,5 & 3,56 & 0,925 \\
\hline 16 & 5 & 5 & 100 & 0 & 0 & 0 & 834,03 & 43,0 & 3,56 & 0,920 \\
\hline 17 & 5 & 5 & 100 & 0 & 0 & 0 & 842,35 & 44,2 & 3,81 & 0,920 \\
\hline 18 & 5 & 5 & 100 & 0 & 0 & 0 & 812,22 & 43,8 & 3,81 & 0,920 \\
\hline 19 & 5 & 5 & 100 & 0 & 0 & 0 & 772,6 & 44,2 & 3,82 & 0,923 \\
\hline
\end{tabular}

a) $\mathrm{x}_{1}=([\mathrm{P}]-5) / 3 ; \mathrm{x}_{2}=([\mathrm{A}]-5) / 3 ; \mathrm{x}_{3}=([\mathrm{G}]-100) / 60$

\subsection{1 - Firmeza}

Os modelos lineares e quadráticos foram ajustados dos resultados de firmeza apresentados na Tabela 2

O modelo quadrático (equação 1), mostrou ser superior ao modelo linear, visto o termo $\mathrm{x}_{1} \mathrm{x}_{2}$ ser significativo. Com exceção do termo quadrático $\mathrm{x}_{3}{ }^{2}$ significativo, todos os demais termos quadráticos e as interações $\mathrm{x}_{1} \mathrm{x}_{3}$ e $\mathrm{x}_{2} \mathrm{x}_{3}$ foram não significativos ao nível de $95 \%$ de probabilidade.

$$
\begin{aligned}
& \hat{y}=840,8+373,2 x_{1}+323,0 x_{2}-7,5 x_{3}-8,8 x_{1}{ }^{2}-41,8 x_{2}{ }^{2}
\end{aligned}
$$

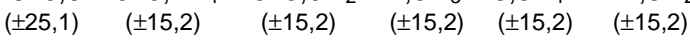

$$
\begin{aligned}
& -80,4 x_{3}^{2}+118,5 x_{1} x_{2}-43,5 x_{1} x_{3}+5,89 x_{2} x_{3} \\
& \begin{array}{llll}
( \pm 15,2) & ( \pm 19,8) & ( \pm 19,8) & ( \pm 19,8)
\end{array}
\end{aligned}
$$

Os resultados obtidos da ANOVA confirmam os ter$\operatorname{mos} \mathrm{x}_{1}, \mathrm{x}_{2}$ e $\mathrm{x}_{1} \mathrm{x}_{2}$ estatisticamente significativos da equa- ção 1. Assim, o modelo contendo somente os termos significativos pode ser descrito da seguinte forma:

$\hat{y}=840,8+373,2 x_{1}+323,0 x_{2}-80,4 x_{3}^{2}+118,5 x_{1} x_{2} \quad$ (Equação 2)

A Figura 2 mostra o gráfico dos valores previstos pela equação 1 contra os resultados experimentais correspondentes. Os pontos são distribuídos ao acaso próximos da linha, demonstrando excelente concordância e que o modelo não tem falta de ajuste significativo.

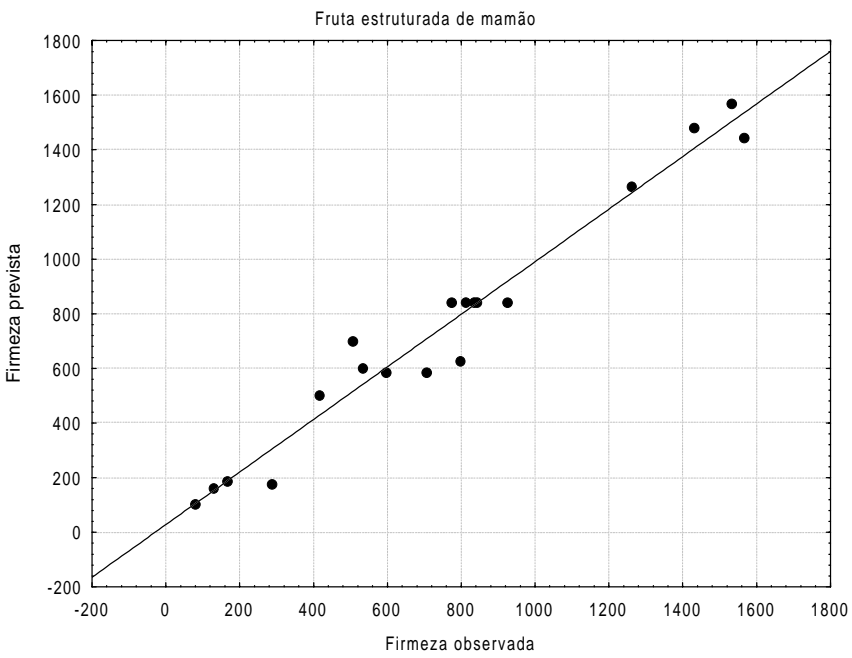

FIGURA 2. Valores de firmeza determinados experimentalmente versus valores previstos pelo modelo quadrático da equação 1. A linha diagonal representa concordância exata.

A Figura 3 mostra as superfícies de respostas para firmeza em função das concentrações codificadas de pectina e alginato.

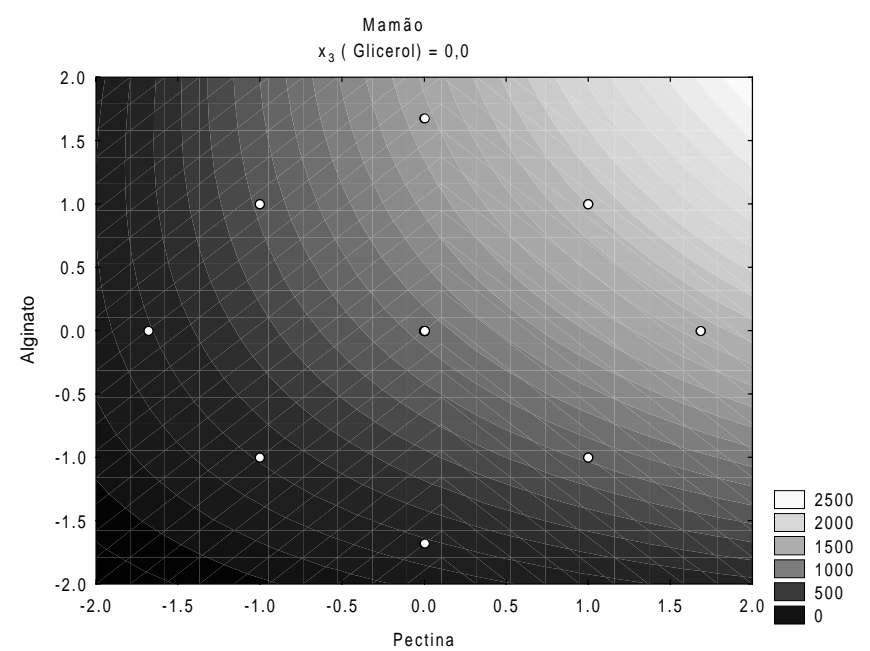

FIGURA 3. Superfície de respostas para fruta estruturada de polpa concentrada de mamão em função das concentrações de pectina $\left(\mathrm{x}_{1}\right)$ e alginato $\left(\mathrm{x}_{2}\right)$. 
As linhas de contorno no alto da superficie, correspondem aos experimentos 4 e 8 da Tabela 2, e mostram que os valores de firmeza, previstos pelo modelo, aumentam sensivelmente com aumento das concentrações de pectina e alginato. Este aumento é mais sensível quando os aumentos em pectina e alginato são iguais. Isto é consistente com os valores dos coeficientes de $x_{1}$ e $x_{2}$ que são positivos e quase iguais, e o valor positivo do coeficiente do termo $\mathrm{x}_{1} \mathrm{x}_{2}$. A interação $\mathrm{x}_{1} \mathrm{x}_{2}$ significativa confirma a existência de sinergismo entre os hidrocolóides conforme relatado por MANCINI \& MCHUGH [11]. De acordo com o modelo matemático, o termo em $\mathrm{x}_{3}$ quadrado, indica que o nivel de glicerol encontra-se na região máxima e qualquer variação no seu teor, poderá diminuir a firmeza das frutas estruturadas de polpa de mamão concentrada.

De modo geral, os géis obtidos de misturas de $8 \mathrm{~g} / \mathrm{kg}$ de pectina e $8 \mathrm{~g} / \mathrm{kg}$ de alginato apresentaram firmeza elevada, acima de $1,4 \mathrm{~kg}$, independente da concentração de glicerol. Foi verificado que a firmeza aumentou 4,4 vezes quando a pectina $\left(\mathrm{x}_{1}\right)$ passa do nivel mais baixo $(-1)$ para o nivel mais alto $(+1)$, mantendo-se os fatores $\mathrm{x}_{2}$ e $\mathrm{x}_{3}$ constantes. $\mathrm{O}$ alginato teve influência semelhante, sendo verificado um aumento médio de 4 vezes na firmeza das frutas estruturadas.

\subsection{2 - pH}

Os valores ajustados dos modelos linear e quadrático para o pH dos géis de mamão são apresentados na Tabela 2. Não foi verificado qualquer termo significativo em ambos modelos. Isto pode ser explicado comparando-se a média e desvio padrão $(3,57 \pm 0,08)$ dos experimentos $1-14$, e os valores $(3,71 \pm 0,14)$ obtidos para as cinco repetições do ponto central (15-19). O desvio padrão para os pontos centrais é maior do que o verificado nos experimentos 1-14, nos quais os niveis dos fatores $\mathrm{x}_{1}, \mathrm{x}_{2}$ e $\mathrm{x}_{3}$ variam consideravelmente. Desta forma, o desvio padrão para o delineamento fatorial e estrela pode ser explicado unicamente pelo erro experimental.

\subsection{3 - Sólidos solúveis}

Os resultados do teor de sólidos solúveis ( $\left.{ }^{\circ} \mathrm{Brix}\right)$ podem ser interpretados da mesma maneira que os valores de $\mathrm{pH}$. Os modelos linear e quadrático não apresentaram termos significativos, embora ambos não tenham apresentado evidências de falta de ajuste. A média e desvio padrão para as repetições foram $43,74 \pm 0,51$, muito similares aos valores dos experimen-

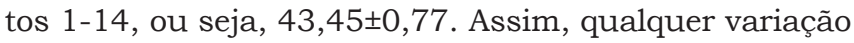
nos valores de sólidos solúveis pode ser atribuída somente ao erro experimental.

\subsection{4 - Atividade de água}

Ao contrário dos sólidos solúveis e do $\mathrm{pH}$, os valores de atividade de água foram bastante sensiveis às mudanças nos niveis dos fatores. Os modelos linear e quadrático apresentaram falta de ajuste significativa, mostrando que estes modelos simplificados não são suficientes para descrever as variações na atividade de água. A média e desvio padrão, 0,923 $\pm 0,0131$, para os experimentos 1-14 e 0,923 $\pm 0,0025$, para a repetição do ponto central mostram que a atividade de água varia mais quando se varia os niveis dos fatores do que para as replicatas dos experimentos. $\mathrm{O} \mathrm{F}_{\text {calculado }}=27,46$ obtido da razão $\left(0,0131^{2} / 0,0025^{2}\right)$ é maior do que o $\mathrm{F}_{\text {tabelado }}=5,87$ para 14 e 4 graus de liberdade ao nivel de $95 \%$ de confiança, indicando diferentes variações populacionais para estes dois grupos de experimentos. No entanto, a dependência da atividade de água em termos das concentrações de pectina, alginato e glicerol é muito complexo para ser explicado pelos modelos usualmente empregados na MSR.

O glicerol não contribuiu na redução da atividade de água dos géis de polpa concentrada de mamão para níveis de umidade intermediária. Foi verificado valor de atividade de água de 0,907, na concentração máxima de glicerol (200g/kg) (Tabela 2), provavelmente devido o elevado teor de água presente na polpa concentrada. Foi observado sinérese e pegajosidade na superficie das frutas estruturadas de polpa concentrada de mamão. As frutas estruturadas submetidas à secagem a $60^{\circ} \mathrm{C}$ sob vácuo $\left(0,8 \mathrm{kgf} / \mathrm{cm}^{2}\right)$ apresentaram teor de umidade reduzido $(9,4 \%)$, e 0,859 de atividade de água, valor dentro da faixa de 0,650 a 0,900 de atividade de água estabelecida para alimentos de umidade intermediária por CHIRIFE \& BUERA [6]. A secagem também favoreceu o ressecamento da película externa, contribuindo para formar um tipo de casca e melhorar a textura final do produto. A aceitação sensorial das frutas estruturadas desidratadas será tratada em próxima publicação.

\section{2 - Confirmação do modelo matemático propos- to para firmeza}

Na Tabela 3 estão listados os valores de concentração de pectina, alginato e glicerol e respectivos valores de firmeza estimados do modelo quadrático (equação 1) para frutas estruturadas de polpa concentrada de mamão.

TABELA 3. Valores das variáveis otimizadas, previstas pela equação 1 para produção de fruta estruturada de polpa de mamão concentrada, e respectivos valores de firmeza

\begin{tabular}{cccc}
\hline $\begin{array}{c}\text { Pectina }\left(\mathrm{x}_{1}\right) \\
\mathrm{em} \mathrm{g} / \mathrm{kg}\end{array}$ & $\begin{array}{c}\text { Alginato }\left(\mathrm{x}_{2}\right) \\
\text { em g/kg }\end{array}$ & $\begin{array}{c}\text { Glicerol }\left(\mathrm{x}_{3}\right) \\
\text { em g/kg }\end{array}$ & $\begin{array}{c}\text { Firmeza calculada } \\
(\mathrm{g})\end{array}$ \\
\hline 7,0 & 7,0 & 100 & 1338 \\
7,5 & 7,5 & 100 & 1465 \\
9,0 & 5,0 & 100 & 1321 \\
\hline
\end{tabular}

Para confirmar as previsões do modelo matemático proposto foram realizados experimentos usando a menor concentração de pectina $(7 \mathrm{~g} / \mathrm{kg})$ e de alginato $(7 \mathrm{~g} / \mathrm{kg})$, que correspondem a firmeza de $1,3 \mathrm{~kg}$, utilizando-se teor elevado de polpa de mamão concentrada $(672 \mathrm{~g} / \mathrm{kg})$ e quantidade mínima de sacarose $(364 \mathrm{~g} / \mathrm{kg})$. O glicerol foi mantido no nivel intermediário $(100 \mathrm{~g} / \mathrm{kg})$ por ter pro- 
porcionado aumento de 34\% no teor de sólidos solúveis da polpa concentrada, e redução na atividade de água na ordem de $7 \%$. As frutas estruturadas assim obtidas, apresentaram firmeza elevada $(3,1 \mathrm{~kg})$, cerca de 2,4 vezes maior do que a prevista pelo modelo. Esse resultado, apesar de animador, não confirma o modelo, que só pode ser usado para fazer previsões de experimentos sob as mesmas condições. O valor 2,4 vezes maior indica que os efeitos de outros fatores no valor da firmeza devem ser investigados. Por exemplo, a variação de $\mathrm{pH}$ entre a polpa utilizada nos experimentos de otimização ( $\mathrm{pH} 4,00)$, e a polpa utilizada nos experimentos para confirmação do modelo $(\mathrm{pH} \mathrm{4,50)}$ pode ter contribuído para produzir um valor de firmeza mais alto. Isso está de acordo com os resultados de KALENTUC, NUSSINOVITCH \& PELEG [10], ou seja, polpas menos ácidas produzem géis mais firmes.

\section{4 - CONCLUSÕES}

Os resultados obtidos mostram que há boa perspectiva para produção de fruta estruturada com teor elevado de polpa concentrada de mamão $(672 \mathrm{~g} / \mathrm{kg})$ e quantidade mínima de sacarose $(364 \mathrm{~g} / \mathrm{kg})$.

A adição de glicerol incrementou de 15 a $60 \%$ o teor de sólidos solúveis da polpa de mamão concentrada, possibilitando redução na quantidade de sacarose adicionada e, conseqüentemente, do aporte calórico das frutas estruturadas assim produzidas.

O glicerol utilizado como supressor de atividade de água não reduziu o teor de água livre das frutas estruturadas para niveis de umidade intermediária. Foi verificado valor de atividade de água de 0,907, mesmo na concentração máxima $(200 \mathrm{~g} / \mathrm{kg})$.

$\mathrm{O}$ pH é um parâmetro importante no processo de estruturação da polpa de mamão concentrada. Variação no $\mathrm{pH}$ da polpa de 4,00 para 4,5, pode promover alteração na firmeza do produto.

Os resultados obtidos do planejamento "central composto" indicam que as variáveis independentes: pectina $\left(\mathrm{x}_{1}\right)$ e alginato $\left(\mathrm{x}_{2}\right)$ devem ser consideradas estatisticamente significativas com o objetivo de aumentar a firmeza dos produtos.

$\mathrm{O}$ modelo matemático evidenciou a variável $\mathrm{x}_{3}$ quadrática significativa, indicando que o nível de glicerol encontra-se na região máxima e qualquer variação no seu teor, poderá diminuir a firmeza das frutas estruturadas de polpa de mamão concentrada.

A secagem a $60^{\circ} \mathrm{C} / 6 \mathrm{~h}$ sob vácuo $\left(0,8 \mathrm{kgf} / \mathrm{cm}^{2}\right)$ das frutas estruturadas promoveu redução no teor de umidade para niveis de umidade intermediária $(\mathrm{Aa}=0,859)$. A secagem também favoreceu o ressecamento da película externa, contribuindo para formar um tipo de casca e melhorar a textura final do produto.

Experimentalmente, verificou-se que as frutas estruturadas produzidas de acordo com as condições otimizadas para as variáveis pectina $(7 \mathrm{~g} / \mathrm{kg})$ e alginato $(7 \mathrm{~g} / \mathrm{kg})$ eram 2,4 vezes mais firmes que o previsto pelo modelo quadrático. A provável explicação seria a variação de $\mathrm{pH}$ entre a polpa concentrada de mamão utilizada nos experimentos de otimização ( $\mathrm{pH} 4,00)$, e a utilizada nos experimentos para confirmação do modelo ( $\mathrm{pH} 4,50)$.

As frutas estruturadas e desidratadas apresentam potencial de consumo na forma que se apresenta, como um confeito, similarmente às barras de frutas (fruit bar) também conhecidas como "couro" de frutas (leather fruit) ou também podem ser utilizadas na formulação de produtos de confeitaria ou alimentos congelados.

\section{5 - REFERÊNCIAS BIBLIOGRÁFICAS}

[1] AGRIANUAL: anuário da agricultura brasileira. São Paulo: FNP Consultoria e Agroinformático, 2003. p. 378386.

[2] AMERINE, M.A.; PANGBORN, R.N.; ROESSLER, E.B. Principle of sensory evaluation of food. New York: Academic Press, 1965. p. 366-373.

[3] BARROS NETO, B.; SCARMINIO, I.S.; BRUNS, R.E. Como fazer experimentos: Pesquisa e desenolvimento na ciência e na indústria. Campinas: UNICAMP, 2002. $401 \mathrm{p}$.

[4] BELLARD, F.B. Utilização de alginato de sódio na produção de um gel estruturado de suco de maracujá (Passiflora edulis S). Campinas, 1995, 60p. Dissertação (Mestrado em Tecnologia de Alimentos), Faculdade de Engenharia de Alimentos, Universidade Estadual de Campinas (UNICAMP).

[5] BOX, G.E.P.; HUNTER, H.G.; HUNTER, J.S. Statistics for experiments. New York: John Wiley Co., 1978. p. 306-351, 501-539.

[6] CHIRIFE J.; BUERA M.D.P. Water activity, glass transition and microbial stability in concentraded/semimois food systems. J. Food Sci. v.59, n.5, p.925-927, 1994.

[7] FIZMAN, S.M.; DURAN, L. Effect of fruit pulps and sucrose on the compression response of different polysaccharides gel systems. Carbohydrate Polymers, v.17, n.1, p.11-17, 1992.

[8] FREITAS, S.M.L. Utilização de alginato de sódio em texturizados de suco misto de laranja e cenoura de valor energético reduzido. Campinas, 1999, 110p. Dissertação (Mestrado em Tecnologia de Alimentos), Faculdade de Engenharia de Alimentos, Universidade Estadual de Campinas (UNICAMP).

[9] INSTITUTO ADOLFO LUTZ. Normas Analiticas do Instituto Adolfo Lutz. 3 ed. v.1: Métodos físicos, químicos para análise de alimentos. São Paulo. 1985.

[10] KAlentuC, G.; NUSSinovitCh, A.; PElEG, M. Alginate texturization of highly acid fruit pulps and juices. J. Food Sci., v. 55, n. 6, p. 1759-1761, 1990.

[11] MANCINI, F.; McHUGH, T.H. Fruit-alginate interactions in novel restructured products. Nahrung-Food, v. 44, n. 3, p. 152-157, 2000.

[12] MARTIN, Z.J.; NISIDA, A.L.A.C.; MEDINA, J.C.; BALDINI, V.L. Processamento: produtos, características e utilização. In: MEDINA, J.C. et al. (Ed.) Mamão: cultura, matéria-prima, processamento e aspectos econômicos. Campinas: ITAL, 1995. cap.3, p. 255334. (Série Frutas Tropicais, 7).

[13] MOUQUET, C.; AYMARD, C.;GUILBERT, S.; CUVELIER, G.; LAUNAY, B. Influence of incial $\mathrm{pH}$ on gelation kinetics of texturized passion fruit pulps. Lebens. Wiss. Tecnol., v. 30, n. 2, p. 129-134, 1997. 
[14] MOUQUET, C.; DUMA, J.C.; GUILBERT, S. Texturization of sweetened mango pulp: optimization using response surface methodology. J. Food Sci., v. 57, n. 6, p. 1395-1400, 1992.

[15] NUSSINOVITCH, A; PELEG, M. Mechanical properties of a raspberry product texturized with alginate. J. Food Proc. and Pres., v. 14, n. 4, p. 267-278, 1990.

[16] NUSSINOVITCH, A; KOPELMAN, I.J.; MIZRAHI, S. Mechanical properties of composite fruit products based on hiydrocolloid gel, fruit pulp and sugar. Lebens. Wiss. Tecnol., v. 24, n., p. 214-217, 1991a.

[17] NUSSINOVITCH, A; KOPELMAN, I.J.; MIZRAHI, S. Modeling the combined effect of fruit pulp and sugar and gum on some mechanical parameters of agar and alginate gel. Lebens. Wiss. Tecnol., v. 24, n., p. 513517, $1991 \mathrm{~b}$.

[18] OWEN, S.R.; TUNG, M.A. Cutting resistance of a restructured fruit bar as incluenced by water activity.
J. Food Text., v. 22, n. 2, p. 191-199, 1991.

[19] PELAEZ, C.; KAREL, M. Improved method for preparation of fruit simulating alginate gels. J. Food Proc. Pres., v. 5, n. 1, p. 63-81, 1981.

[20] STABLE MICRO SYSTEMS. User Manual. Texture Analyser. Model TA-XT2i, Godalming, version 6.10 e 7.10, Agosto de 1997, 87p.

[21] VIJAYANAND, P.; YADAV, A. R.; BALASUBRAMANYAM, N.; NARASIMHAM, P. Storage stability of guava fruit bar prepared using a new process. Lebens. Wiss. Tecnol., v. 33, n. 1, p. 132-137, 2000.

\section{6 - AGRADECIMENTOS}

Os autores RKG e JMA agradecem à Fundação de Amparo à Pesquisa do Estado de São Paulo (FAPESP) pela concessão de Bolsa de Pós-Doutorado e Auxílio à Pesquisa. 